\title{
IGUALDAD DE OPORTUNIDADES Y POLITIICA EDUCATIVA
}

\author{
JUAN CARLOS TEDESCO \\ Instituto Internacional de Planificación de la Educación, Filial da Unesco \\ Buenos Aires, Argentina \\ j.tedesco@iipe-buenosaires.org.ar
}

\section{RESUMEN}

El texto está dividido en dos secciones. La primera está destinada a analizar la hipótesis según la cual los diagnósticos y las respuestas a los cambios sociales actuales movilizan no sólo las competencias científicas y técnicas de los sujetos y de los grupos sociales sino también sus sistemas básicos de valores. La decisión ética es previa a la definición de estrategias políticas. La segunda parte del texto está dedicada a explorar las estrategias políticas más apropiadas para romper el determinismo social de los resultados de aprendizaje. En este sentido, se mencionan tanto las politicas de atención temprana a la niñez como las estrategias de cambio pedagógico, con especial énfasis en lo que se denominan "politicas de subjetividad".

POLITICASDEEDUCACIÓN-CAMBIOSOCIAL - APRENDIZAJE

\section{ABSTRACT}

EQUAL OPPORTUNITIES AND EDUCATIONAL POLICY. This text is divided in two sections. The first one analyzes the hypothesis according to which the diagnoses and responses to the current social changes not only stir the scientific and technical skills of individuals and of social groups, but also their basic systems of value. The ethical decision is prior to the definition of politicalstrategies. The second part of the text explores the most appropriate politicalstrategies to break the social determinism of learning results. In this sense, both policies of early attention to children and of pedagogical change strategies are mentioned, with a special emphasis on the socalled "subjective policies".

EDUCATIONAL POLICY - SOCIAL CHANGE - LEARNING

Texto apresentado no Seminário: Políticas Inclusivas e Políticas Compensatórias na Agenda de Educação, comemorativo dos 40 anos da Fundação Carlos Chagas, realizado em 30.9 e $1^{\circ}$. 10.2004 , em São Paulo. 
La literatura y las prácticas políticas destinadas a analizar y a promover la igualdad de oportunidades a través de la educación son muy amplias, variadas y tienen una larga tradición histórica. ¿Cuál es la razón por la cual, a pesar de estos antecedentes, hoy es necesario volver a analizar este problema? La respuesta a esta pregunta implica aceptar que los profundos cambios que vive actualmente la sociedad, tanto desde el punto de vista económico como político y cultural, nos obligan a revisar nuestras concepciones sobre el problema y nuestras formas de intervención. Esta modificación afecta especialmente la igualdad de oportunidades ya que una de las tendencias más fuertes de la nueva economía es el aumento de las desigualdades y ese aumento está acompañado por tendencias igualmente fuertes a la segmentación espacial y a la fragmentación cultural de la población.

No estamos, en consecuencia, frente a una cuestión secundaria. Al contrario, los análisis sobre la sociedad contemporánea que provienen de sociólogos, economistas, antropólogos, filósofos y educadores muestran que el interrogante principal que abre la evolución de la sociedad contemporánea se refiere, precisamente, a la posibilidad de construir un orden social basado en la justicia y en el reconocimiento de la igualdad básica de los seres humanos. En un contexto de este tipo, nos parece necesario postular que, para avanzar en la comprensión de la relación entre igualdad de oportunidades y educación, no alcanza con un enfoque basado exclusivamente en paradigmas científicos o técnicos. Necesitamos ampliar la mirada e introducir la dimensión ética que nos permita justificar porqué y para qué deseamos construir socialmente una situación donde todos tengan las mismas oportunidades.

La hipótesis sobre la cual se apoya este texto es que los diagnósticos acerca de los actuales cambios sociales, así como las respuestas a dichos cambios, movilizan no sólo conocimientos científicos o posiciones políticas, sino los sistemas básicos de valores de los ciudadanos y de los grupos sociales. No se trata de apelar a los valores en un vacío científico o ideológico-político. Se trata, en cambio, de aceptar que un enfoque basado sólo en el aporte de la ciencia y de la técnica muestra sus límites cuando se trata de optar entre alternativas que el conocimiento o las ideologías disponibles no alcanzan a explicar ni a resolver.

Las limitaciones del enfoque científico-técnico está vinculado están vinculadas a la propia práctica científica. Al respecto, es oportuno recordar el análisis de Beck (200 I), quien nos advierte que en la ciencia actual, primero es 
necesario aplicar las teorías y producir ciertos fenómenos, para luego estudiar sus propiedades y características: "Es preciso producir primero niños probeta, liberar criaturas artificiales genéticamente modificadas y construir reactores, para poder estudiar sus propiedades y características de seguridad". En un contexto de este tipo, la ciencia revela sus límites para dar respuestas a las preguntas por el sentido de nuestras acciones, respuestas que dependen básicamente de la política y de la ética.

El ejercicio del saber social siempre estuvo asociado a opciones éticas, políticas o ideológicas. No se trata, en consecuencia, de perder el rigor científico ni de caer en opciones puramente ideológicas. Como sostuviera Wallerstein (2002), la idea de que el saber científico y el filosófico-humanístico son radicalmente diferentes, como si constituyeran dos formas intelectualmente opuestas de "saber" el mundo - algo que a veces se dio en llamar la tesis de las "dos culturas" -, no sólo se está volviendo inadecuada como explicación de la masiva transición social que atravesamos, sino que incluso se convierte en un obstáculo a la hora de enfrentar la crisis de manera inteligente.

En este marco, el texto que sigue estará dividido en dos grandes secciones. La primera estará destinada a explicitar el desafío ético de la construcción de un orden social basado en la igualdad de oportunidades mientras que la segunda estará destinada a señalar algunas líneas estratégicas en el campo de las políticas educativas, que traduzcan esa voluntad ética en orientaciones para la acción.

\section{LA DIMENSIÓN ÉTICA DEL PROBLEMA}

La nueva economía, o la economía del conocimiento, ha modificado las bases sobre las cuales se asentaban tradicionalmente el Estado de Bienestar, las posibilidades de movilidad social y las distinciones entre las diferentes categorías sociales'. Una de las claves de estos nuevos procesos es el acceso a la educación y al conocimiento. Los trabajadores no calificados y las personas sin competencias para el aprendizaje a lo largo de toda la vida no tendrán posibilidades de obtener empleos decentes. Los cambios en la organización del

I La bibliografía sobre la sociedad del conocimiento y las nuevas desigualdades es muy amplia. Entre otros, pueden verse Castel (1997), Cohen (1998) y Rosanvallon (1995). 
trabajo y en las demandas de calificaciones explican en gran medida la tendencia al aumento de la desigualdad. Dicha tendencia se expresa con mayor intensidad en los países con mayores niveles de desregulación de sus mercados de trabajo y de sus políticas sociales. No es casual por ello que también sea en esos contextos donde se producen los argumentos más claramente orientados a justificar la desigualdad social como un fenómeno natural y legítimo².

Las posibilidades de un orden social basado en la desigualdad natural de los seres humanos han recibido un nuevo impulso a partir del descubrimiento del genoma humano. Al respecto, es oportuno retomar el análisis reciente que Habermas (2002) presentó sobre las consecuencias de la manipulación genética sobre la construcción del orden social. Habermas sostiene que la intervención genética modifica las condiciones a partir de las cuales nos constituimos en sujetos, en nosotros mismos. En el marco de la socialización basada en un capital genético no manipulado, tenemos un principio de libertad que nos permite asumir la responsabilidad sobre nuestra biografía, la reflexión autocrítica y la posibilidad de compensar retrospectivamente la relación asimétrica que existe entre padres e hijos. Los deseos de los padres son siempre susceptibles de contestación en el proceso comunicacional de la socialización. Esta posibilidad autocrítica desaparece o se modifica cuando sabemos que existió una intervención intencional de otros en nuestro capital genético. Llevado al extremo, es posible suponer que el nuevo ser devenido adulto no dispondrá de la posibilidad de instaurar la necesaria simetría de responsabilidades recurriendo a la autorreflexión ética. Con la intervención genética cambian las relaciones de poder entre las personas. El "programador" interviene como protagonista en el interior de la vida de la persona programada, pero sin la posibilidad de ser un "antagonista", como es el caso de la constitución del sujeto a través del proceso de socialización. Es cierto que la sociedad se caracteriza por la desigualdad, la opresión despótica, la privación de derechos, la explotación económica. Pero sólo nos podemos revelar contra estas situaciones si sabemos que pueden ser diferentes. El paternalismo generado por la manipulación genética es totalmente diferente al paternalismo conocido hasta ahora.

Las consecuencias abiertas por las posibilidades de manipulación del ca-

2 Ver, al respecto, el debate que provocó en EEUU la publicación del libro de Herrnstein y Murria (1994). 
pital genético de los hijos nos coloca ante un fenómeno paradójico. En el momento en el cual la familia se democratiza, que las relaciones entre padres e hijos han alcanzado un grado muy alto de simetría y en el cual los adultos han perdido capacidad para imponer determinadas visiones del mundo a las nuevas generaciones, se abre la posibilidad de ejercer formas de poder extremas, irreversibles, no sujetas a ningún tipo de diálogo, de comunicación y de intercambio.

Las consecuencias de estos posibles desarrollos sobre la educación en un sentido general son inéditas. No se trata sólo de la posibilidad de establecer una oferta educativa adaptada a supuestos perfiles genéticos que impidan procesos de movilidad social, de cambios en destinos prefijados de antemano, lo cual es de por sí sumamente grave socialmente. Mucho más grave aun es la perspectiva de una ruptura en las condiciones sobre las cuales se apoya nuestra idea de autonomía personal, de responsabilidad sobre mi nuestra propia historia $y$, en definitiva, sobre la base moral en la cual se asienta la sociedad.

Sin embargo, sería utópico pensar que un modelo de exclusión y de desigualdad como el que prefiguran estos análisis pueda ser sostenido en el tiempo sin altos niveles de conflictividad y de crítica por parte no sólo de los excluidos sino de sectores de población incluida, pero dotada de un fuerte sentido de responsabilidad social. En una reflexión relativamente reciente, Lester Thurow llevó este razonamiento de la sustentabilidad al límite de sus posibilidades. Refiriéndose a EEUU como el modelo dominante de desarrollo de la economía y la sociedad del conocimiento, Thurow mostró cómo la dinámica económica permitirá que una parte de la fuerza de trabajo, con las habilidades necesarias para integrarse a la nueva economía, participará de los beneficios del crecimiento económico, dejando atrás y afuera al resto de la fuerza de trabajo.

El problema no es que este modelo no logre funcionar sino que, al contrario, puede hacerlo.

Los problemas con el modelo de desarrollo económico de enclave no son económicos. Podría funcionar para los norteamericanos capacitados tal como funciona para los ingenieros de software en Bangalur, en India. Los problemas ni siquiera son realmente políticos. India es un ejemplo de que en los países pueden coexistir grandes desigualdades internas durante largos períodos de tiempo sin que estallen políticamente. Los problemas son básicamente morales. ¿Vive uno en una buena sociedad si esa sociedad permite de manera consciente que una gran parte de sus 
ciudadanos se vaya salga del primer mundo y se convierta efectivamente en trabajadores que ganan salarios del tercer mundo? (Thurow, 2000, p.327)

Esta pregunta, que Thurow reduce al ámbito nacional de los EEUU, debe ser entendida a escala planetaria. ¿Podemos aceptar vivir en un mundo que deje afuera un porcentaje muy importante de la población? Este es el dilema. Sólo si no aceptamos esta situación es que tiene sentido explorar las alternativas técnicamente más eficaces para lograr el objetivo ético-político de una sociedad justa.

\section{LA DIMENSIÓN TÉCNICO-POLÍTICA}

La voluntad ética de construir una sociedad basada en los principios de justicia y equidad tiene que traducirse en acciones que pongan de manifiesto que alcanzar ese objetivo es posible. En este sentido, la última década ha sido un período donde se ha producido un fenómeno del cual es preciso sacar todas las conclusiones y aprendizajes necesarios: a pesar del aumento en la inversión educativa en educación y a pesar de los procesos de reforma que la mayor parte de los países han desarrollado, las desigualdades educativas persisten $y$, en algunos casos, han aumentado.

El Ministro de Educación de Francia, para citar un caso elocuente de este fenómeno, resumía recientemente la situación de su país donde, entre 1990 y el año 2000 se incrementó el presupuesto público destinado a educación en un $25 \%$, aumentó el número de docentes entre el 2\% y el 7,8\% para la enseñanza primaria y secundaria respectivamente, descendió disminuyó el número de alumnos por factores demográficos en 423.000 para primaria y 1 30.000 para secundaria y aumentó el gasto por alumno entre un $94 \%$ y un $74 \%$ para cada nivel respectivamente. A pesar de estas condiciones objetivamente más favorables, los resultados educativos no han mejorado y siguen asociados directamente al origen social de los alumnos (Ferry, 2003).

No es éste el lugar para un análisis detallado de los factores que explican los resultados escolares, pero es importante destacar que existe un consenso bastante general en asignar la responsabilidad por los logros de aprendizaje de los alumnos a dos factores principales: el entorno familiar y la efectividad de la escuela. Brunner resumió los resultados de los principales estudios sobre lo- 
gros de aprendizaje y mostró que para los países desarrollados, alrededor del $80 \%$ de los logros de aprendizaje son explicados por variables ligadas al entorno familiar y sólo el $20 \%$ se explica por la acción de la escuela 3 .

Esta constatación empírica está en la base de las corrientes de pensamiento político que sostienen la necesidad de otorgar una prioridad muy fuerte a las intervenciones antes del ingreso a la escuela y a las intervenciones escolares más eficaces en romper el determinismo impuesto por las condiciones sociales. Veamos a continuación estos dos problemas por separado.

\section{La atención temprana}

Los datos más recientes muestran que existen serios riesgos de un incremento en las tendencias a la reproducción de la pobreza y de las desigualdades sociales. La precariedad en los contratos de trabajo está aumentando y afecta fundamentalmente a niños y jóvenes. Si se agregan a esos fenómenos la tendencia a la selección marital en términos de educación, es muy probable esperar que la polarización social aumente y que la pobreza y la precariedad tienda a reproducirse en las mismas familias. En el contexto europeo, los países que muestran los mejores índices de equidad social son los países escandinavos, que se caracterizan precisamente por sus políticas familiares, que permiten a las madres tener acceso al mercado de trabajo mientras los hijos son atendidos desde muy temprano por instituciones de cuidado infantil ${ }^{4}$.

Los análisis acerca del proceso de reproducción de las desigualdades permiten señalar que para romper el círculo vicioso de padres pobres hijos pobres, es fundamental intervenir en el momento donde en que se produce la formación básica del capital cognitivo de las personas. Esto significa invertir en las familias y en la primera infancia. Existen numerosas evidencias que indican

3 Brunner (2002) analiza los resultados de II investigaciones, llevadas a cabo entre 1996 y el año 2000. Incluye desde los estudios clásicos de Coleman y Jencks hasta los más recientes de Luyten, Sheerens y Bosker y Marzano.

4 Un muy interesante trabajo que demuestra la importancia de la atención temprana puede verse en Esping-Andersen (2003). 
el escaso poder compensador de las desigualdades que tiene la educación formal, si interviene una vez que las desigualdades ya han sido creadas.

Desde este punto de vista, el análisis tradicional acerca del vínculo entre educación y equidad social requiere una reformulación importante. Siempre se ha insistido en la idea según la cual la educación es un factor crucial de equidad social y las evidencias empíricas que avalan esta hipótesis son numerosas y bien conocidas por todos. Pero las situaciones sociales creadas por la nueva economía sugieren la necesidad de postular la relación inversa y preguntarse icuánta equidad social es necesaria para que haya una educación exitosa?

Esta situación sugiere que una parte fundamental de la explicación del problema de las dificultades para elevar los resultados de la acción escolar de los alumnos de familias desfavorecidas está vinculada con las condiciones con las cuales los alumnos ingresan en la escuela. Estas condiciones se refieren a dos tipos de factores distintos: (a) un desarrollo cognitivo básico, que se produce en los primeros años de vida y está vinculado a una sana estimulación afectiva, buena alimentación y condiciones sanitarias adecuadas, y (b) una socialización primaria adecuada mediante la cual los niños adquieren los rudimentos de un marco básico que les permita incorporarse a una institución especializada distinta a de la familia, como la escuela.

Las informaciones disponibles sobre movilidad intergeneracional en países desarrollados indica indican que en el largo plazo no se han producido modificaciones importantes en términos de reproducción hereditaria de la estructura social, con la excepción de los países nórdicos, donde la pobreza infantil es prácticamente inexistente. En este sentido, los datos indican que resulta mucho más importante el capital social y cultural de las familias que su nivel de ingresos. Las políticas al respecto deberían, por ello, atacar las desigualdades en términos de recursos culturales disponibles en las familias, que favorezcan un proceso de socialización primaria destinado a promover un adecuado desarrollo cognitivo básico (Esping-Andersen, 2003, p. I 45-146).

\section{La acción escolar}

Si bien el impacto de las acciones propiamente escolares en la igualación de oportunidades es significativamente menor que las acciones destinadas a garantizar las bases del desarrollo cognitivo antes de ingresar a la escuela, no 
es legítimo desconocer la importancia y la necesidad de actuar también en esta fase del desarrollo personal, particularmente si tenemos en cuenta la situación de las generaciones que ya están dentro del sistema escolar y que no tendrán oportunidad de recibir una mejor atención temprana.

En este contexto, nos limitaremos a destacar dos grandes líneas de análisis: a. la que concierne a la organización de la acción escolar, donde el debate gira alrededor de las políticas destinadas a modificar la oferta escolar o a modificar la demanda; y b. la que concierne a la acción pedagógica propiamente dicha, donde la discusión gira alrededor de la prioridad a los insumos materiales del aprendizaje o a los factores subjetivos de los actores del proceso de enseñanza-aprendizaje.

La reforma educativa: entre la oferta y la demanda

Los sistemas educativos tradicionales fueron diseñados a partir del principio según el cual lo importante era controlar la oferta. La escuela - y también la TV general - estaba basada en ofrecer a todos un mismo producto y esta oferta tenía, por eso, un fuerte poder homogeneizador. En esta lógica y en este poder se expresaba la voluntad hegemónica de los sectores dominantes. Los nuevos mecanismos culturales de la sociedad y la economía del conocimiento, en cambio, se basan mucho más en la lógica de la demanda. Internet, la TV por cable (y la escuela basada en los mecanismos de responder a las demandas del "alumno-cliente"), invierten el esquema existente en el capitalismo tradicional y, en ese sentido, más que homogeneizar tienden a fragmentar, a diferenciar, a separar.

Aparentemente, esta adecuación a las demandas estaría respondiendo a los reclamos de respeto a las diferencias, a las identidades y a las opciones individuales. Sin embargo, algunas investigaciones de estos temas han alertado acerca de los riesgos antidemocráticos de esta dinámica cultural basada exclusivamente en la demanda de los usuarios. Según este planteo, para formular una demanda es necesario dominar los códigos de acceso al mundo. Al contrario de lo que sostiene el discurso actualmente dominante, la emancipación, el desarrollo personal, la libertad, pasan primero por la oferta, pues es ella la que permite constituir los marcos de referencia a partir de los cuales se podrá expresar, ulteriormente, la demanda. Una de las modalidades más importantes de la dominación sociocultural consiste, precisamente, en no pedir más que 
lo que uno ya tiene. La simple adecuación a la demanda, en última instancia, implica reforzar la dominación $n^{5}$.

En esta misma línea de análisis se pueden retomar las reflexiones de Rifkin (200I) acerca del nuevo capitalismo como un capitalismo del acceso. Según su análisis, el nuevo capitalismo se caracteriza por incorporar plenamente la esfera cultural dentro de relaciones de tipo mercantil: "la comercialización de los recursos culturales incluyendo los ritos, el arte, los festivales, los movimientos sociales, la actividad espiritual y de solidaridad y el compromiso cívico, todo adopta la forma de pago por el entretenimiento y la diversión personal". En este sentido, los productos culturales ya no valen por sí mismos, no tienen una existencia permanente, pierden su carácter de productos endógenos, pueden ser producidos en cualquier lugar y adquiridos en otros y, lo más importante desde nuestro punto de vista, no son transmitidos de generación en generación sino comprados y vendidos en un mercado donde predomina el poder de compra de cada actor o sector social. La transmisión cultural ha perdido ese carácter conservador, autoritario, reproductor, que tenía en el capitalismo industrial y, obviamente, no se trata de reclamar su retorno. Pero las nuevas formas de producción y distribución cultural tienen un fuerte potencial desestabilizador que no garantiza mayores niveles de libertad y de desarrollo personal sino, según los contextos sociales en los cuales nos movamos, mayores niveles de dependencia o de anomia.

Esta caracterización de la dinámica cultural de la sociedad del conocimiento nos permite analizar las políticas de autonomía a los establecimientos escolares, las estrategias de cambio curricular, el financiamiento de la demanda educativa, la formación docente etc., en el marco de la tensión legítima que debe existir entre el respeto a la diversidad por un lado y la necesidad de promover la cohesión social por el otro.

\section{Aprendizaje y politicas de subjetividad}

La experiencia de la última década de reformas educativas también nos ha permitido apreciar que las reformas institucionales son necesarias pero no suficientes para romper los determinismos sociales y culturales de los resultados de aprendizaje. Los estudios al respecto muestran que la efectividad de la

5 Ver, al respecto, los análisis de Wolton (200I) sobre las nuevas tecnologías de la información. 
acción escolar está generalmente asociada a una serie de insumos, entre los cuales se destacan el conocimiento que el docente tiene de su materia, la disponibilidad de textos, el tiempo dedicado al aprendizaje, la alimentación de los alumnos, el tamaño del colegio, la infraestructura escolar etc. Pero si bien hay consenso en reconocer la importancia de estos factores, también se reconoce que intervenir sobre ellos no modifica automáticamente lo que sucede en la sala de clase, en la relación entre el docente y los alumnos. El testimonio del Ministro de Educación de Francia, así como el análisis de los resultados de las evaluaciones internacionales de resultados del aprendizaje, ponen de relieve la necesidad de introducir otras variables tanto en el análisis como en las estrategias políticas de intervención.

La hipótesis de trabajo que deseamos postular consiste en sostener que sin dejar de reconocer la importancia de mejorar los insumos materiales del aprendizaje - es fundamental prestar atención a la dimensión subjetiva de los actores del proceso pedagógico ${ }^{6}$.

Esta valorización de la subjetividad permite recuperar buena parte del debate contemporáneo acerca de lo que se ha dado en llamar las nuevas desigualdades. Dicho debate ha permitido apreciar que mientras las desigualdades tradicionales eran fundamentalmente "inter-categoriales", las nuevas desigualdades son "intra-categoriales". Contemplado desde el punto de vista subjetivo, una de las características más importantes de este fenómeno es que ahora la desigualdad resulta mucho más difícil de aceptar, porque pone en crisis la representación que cada uno tiene de sí mismo. Estas nuevas desigualdades provocan, por ello, un sufrimiento mucho más profundo, porque son percibidas como un fenómeno más personal que socio-económico y estructural (Fitoussi, Rosanvallon, 1997). Paradójicamente, este mayor sufrimiento subjetivo está acompañado por una legitimidad mucho más fuerte de la desigualdad en el plano público y social. En la medida que se privatizan las responsabilidades de la desigualdad social, se vuelven más opacas las responsabilidades públicas.

6 Al respecto, puede ser interesante recuperar algunos de los aportes de los estudios basados en el concepto de resiliencia. Desde este enfoque, no se trata de negar la relevancia de los factores objetivos, sino de distinguir los efectos del traumatismo de los efectos de la representación del traumatismo. Contrariamente a lo que habitualmente se cree, en nuestra cultura los efectos biológicos, por ejemplo, son a menudo reparables debido a la plasticidad de nuestro cerebro, mientras que los efectos atribuibles a la representación subjetiva del trauma - provocada ya sea por el discurso social o académico - pueden ser mucho más difíciles y lentos de restaurar. Ver, por ej. Cyrulnik (200I). 
Reconocer la importancia de la dimensión subjetiva de los fenómenos sociales plantea nuevos problemas a la teoría y a la acción política. Las políticas sociales destinadas a enfrentar los problemas asociados a la pobreza suelen ser políticas de masas, con escasas o nulas posibilidades de personalización. Sólo en contextos donde la pobreza afecta a sectores reducidos de población y donde existe una relativa abundancia de recursos, parece posible plantearse la posibilidad de personalizar las estrategias de intervención. En situaciones donde es preciso atender contingentes muy numerosos con pocos recursos, la tentación por ignorar la dimensión subjetiva del problema es muy fuerte. Sin embargo, nadie puede suponer, bajo el pretexto de la necesidad de atender necesidades masivas, que la subjetividad es menos necesaria en los servicios destinados a sectores de bajos recursos que en los servicios para población de altos recursos.

Los estudios sobre políticas compensatorias en educación, por ejemplo, ponen de en relieve los límites de las estrategias de carácter masivo (lo mismo para todos), pero también son elocuentes en cuanto a mostrar las dificultades que existen para incorporar la dimensión subjetiva en los modelos de gestión de dichas políticas (Organización de los Estados Americanos, 2002).

Dicho en otros términos, estaríamos ante la posibilidad de avanzar en el diseño de políticas de subjetividad, tema muy complejo pero que es preciso comenzar a desarrollar? Según esta línea de análisis, lo que sigue es un intento de identificar aquellos aspectos que aparecen en el trayecto de las personas o de los grupos que logran superar los determinismos sociales y culturales y que tienen vinculación directa con el trabajo pedagógico. Una política educativa que pretendiera asumir el reto de la subjetividad para garantizar igualdad de oportunidades debería - si este análisis fuera válido - hacerse cargo de estas dimensiones.

Sin pretender ser exhaustivos, parece plausible destacar al menos tres aspectos que pueden ser objeto de una política educativa y que han demostrado tener significativa importancia en los casos de experiencias exitosas: a. la capacidad para formular un proyectos; b. la capacidad para elaborar una narrativa acerca de la situación; y c. la confianza por parte de adultos significativos en la capacidad del sujeto para superar la situación adversa. La fertilidad y la

7 La subjetividad no se reduce desde esta perspectiva, a la intimidad de una persona. Según la feliz expresión de Ehrenberg (apud Martucelli, 2002), "la subjetividad se ha transformado en una cuestión colectiva”. Sobre este tema, ver el interesante capítulo que Martucelli dedica a la subjetivad. 
pertinencia de estos tres factores se derivaderivan, en gran parte, del hecho que ya han sido señaladas desde la propia práctica pedagógica como muy importantes para el trabajo educativo.

Proyecto

Existen numerosos testimonios que indican que una de las características de las personas o las comunidades que logran superar las condiciones adversas es que disponen de un proyecto para el futuro. Disponer de un proyecto es muy importante tanto para soportar el trauma como para superarlo. Pero, en un sentido más amplio, ser capaz de elaborar un proyecto es un aspecto central en el proceso de construcción de un sujeto.

La teoría sociológica se ha encargado de mostrar que la capacidad y la posibilidad de elaborar un proyecto están socialmente determinadas. Al respecto, es posible evocar los estudios donde se advierte que la ausencia de proyectos es uno de las los factores más significativos en la caracterización de la pobreza. Esta carencia se ha acentuado en las últimas décadas, como consecuencia de los cambios sociales y económicos, que aumentaron significativamente los niveles de incertidumbre sobre el futuro. "En un mundo en cambio y fuera de control, no existe otro punto de apoyo que el esfuerzo del individuo para transformar las experiencias vividas en construcción de sí como actor", sostuvo Alain Touraine (1997, p.28) para explicar el concepto de sujeto. Pero este proceso de construcción del sujeto (que pasa básicamente por la capacidad de definir un proyecto de vida) requiere apoyos institucionales, particularmente los que brindan la familia y la escuela. Desde esta perspectiva, es posible recuperar los análisis y propuestas que enfatizan la necesidad de concebir la tarea educativa como una tarea de orientación.

Esta función de orientación puede ejercerse a través del fomento de una gran diversidad de actividades, que incluyen desde el manejo de los códigos con los cuales se procesa la información y se expresan las demandas hasta la discusión general acerca de la evolución de la sociedad, de la cultura, de la economía, de las nuevas profesiones y el fomento de la participación en los procesos de toma de decisiones. Pero desde el punto de vista de la subjetividad, particularmente de aquellos que viven situaciones de pobreza y exclusión, el aspecto central de la tarea educativa consiste en plantear las preguntas claves de la identidad personal: ¿Qué quiero ser? ¿en qué creo? ¿cuáles son mis for- 
talezas y mis debilidades? La escuela y los adultos que la ocupan deberían ayudar a los jóvenes a plantearse y contestarse estas preguntas (vide Azevedo, 1999). Un enfoque de este tipo orientaría, sin dudas, una serie de estrategias tanto desde el punto de vista curricular como desde el punto de vista de las estrategias de enseñanza-aprendizaje, la formación docente y la integración de equipos profesionales en las escuelas.

Narrativa

La constitución del sujeto también está asociada a la capacidad de articular en un relato las imágenes y representaciones vinculadas a la trayectoria de vida. Desde el momento que una persona puede relatar lo que le ha sucedido, sus sentimientos y sus interacciones con el medio se modifican. La naturaleza de lo sucedido deja de ser puramente sensorial para transformarse en verbal y dirigida a alguien que no estuvo presente en el momento de la situación. Recomponer el acontecimiento con palabras no sólo modifica las representaciones mentales de la persona y el sentido que él le atribuye a lo sucedido sino que permite establecer vínculos de confianza con nuevos interlocutores (Cyrulnik, 200I).

Fortalecer la capacidad de producir relatos, de establecer nexos lógicos entre distintas representaciones, supone tener un fuerte dominio del código de la lecto-escritura. Desde este punto de vista, la justificación acerca de la prioridad que debe asumir el aprendizaje de la lecto-escritura en las estrategias destinadas a mejorar la calidad de la educación, adquiere un nuevo estímulo y sentido. Alrededor de este eje curricular se articulan una serie de actividades (el teatro, la literatura, la poesía etc.) de alto valor en el trayecto de la superación de los determinismos. En definitiva, se trata de orientar la enseñanza de la lectura y la escritura hacia el objetivo de fortalecer la capacidad de expresar demandas y necesidades y de comprender lo que sucede.

\section{Confianza}

La confianza ha sido objeto de frecuentes análisis en los estudios sobre el papel que juegan las expectativas del docente sobre la capacidad de aprendizaje de los alumnos. El clásico estudio de Rosenthal sobre el "efecto Pygmalion" es uno de los más frecuentemente evocados para justificar la importancia que 
reviste la actitud del docente y sus expectativas en la determinación del fracaso o el éxito escolar.

Pero los estudios y la experiencia indican que la confianza es un objeto difícil de administrar. Las representaciones que tanto alumnos como maestros tienen de sí mismos o de los otros son objetos construidos en forma lenta y sólida. Al contrario de agresiones intensas pero momentáneas, que suelen dejar menos impacto en la memoria, las agresiones producidas por estigmas sociales son durables y difíciles de modificar. Exagerando un poco nuestra disponibilidad de conocimientos, podríamos decir que conocemos relativamente bien el contenido y los procesos de construcción social de las representaciones, pero sabemos muy poco o nada acerca de cómo modificarlas. La pedagogía enfrenta aquí una de sus barreras más serias, ya que la modificación de estos estigmas implica un trabajo "contra-cultural". En este sentido, construir una escuela y unos docentes capaces de promover trayectorias de aprendizaje que superen los determinismos sociales implica adoptar un enfoque político-educativo con claros compromisos con la equidad social. El punto central de la discusión, sin embargo, es cómo traducir dicho compromiso en actitudes y procedimientos pedagógicos técnicamente eficaces. Sabemos, en todo caso, que la confianza y la modificación de representaciones pasa por dimensiones de la personalidad que van mucho más allá de la dimensión cognitiva. La información es necesaria, pero sólo si se trabaja con la afectividad será posible modificar estereotipos y prejuicios.

\section{BIBLIOGRAFÍA}

AZEVEDO, J. Vôos de borboleta: escola, trabalho e profissão. Porto: Asa, 1999.

BECK, U. La Sociedad del riesgo global. Madrid: Siglo XXI de España, 200I .

BRUNNER, J. J. Límites de la lectura periodística de resultados educacionales. In: IIPEUnesco. Evaluar las evaluaciones: una mirada política de las evaluaciones de la calidad educativa. Buenos Aires: IIPE, 2003, p. 67-84. [El trabajo presentado en el Seminario Internacional Evaluaciones de los sistemas educativos, en Santiago de Chile, dec, 2002.]

CASTEL, R. Metamorfosis de la cuestión social: una crónica del salariado. Buenos Aires: Paidós, 1997.

CYRULNIK, B. Les Villains petits canards. Paris: Odile Jacob, 200I. 
COHEN, D. Riqueza del mundo, pobreza de las naciones. Buenos Aires: Fondo de Cultura Económica, 1998.

ESPING-ANDERSEN, G. Against social inheritance. In: EHRENBERG. Progressive futures: new ideas for the centre-left. London: Policy Network, 2003. p. I27-I 52.

FERRY, L. Lettre à tous ceux qui aiment l'école: pour expliquer les reformes en cours. Paris: O. Jacob, 2003.

FITOUSSI, J.-P.; ROSANVALLON, P. La Nueva era de las desigualdades. Buenos Aires: Manantial, 1997.

HABERMAS, J. L'Avenir de la nature humaine: vers un eugénisme libéral? Paris: Gallimard, 2002.

HERRNSTEIN, R. J.; MURRIA, Ch. The Bell curve: intelligence and class structure in american life. New York: Free Press Paperbacks, 1994.

MARTUCELLI, D. Grammaire de l'individu. Paris: Gallimard, 2002.

ORGANIZACIÓN DE LOS ESTADOS AMERICANOS. Ministerio de Educación, Ciencia y Tecnología. Estrategias sistémicas de atención a la deserción, la repitencia y la sobreedad en escuelas de contextos desfavorecidos: un balance de los años '90 en la Argentina. Buenos Aires, 2002.

RIFKIN, J. El Capitalismo del acceso. Buenos Aires: Paidós, 2001.

ROSANVALLON, P. La Nueva cuestión social. Buenos Aires: Manantial, 1995.

TOURAINE, A. Pourrions-nous vivre ensemble? Egaux et differents. Paris: Fayard, 1997.

THUROW, L. C. Construir riqueza. Buenos Aires: J. Vergara, 2000.

WALLERSTEIN, I. Un Mundo incierto. Buenos Aires: Libros del Zorzal, 2002.

WOLTON, D. Internet iy después? Barcelona, Gedisa, 2001.

Recebido em: agosto 2004

Aprovado para publicação em: agosto 2004 